\title{
Building connectedness and confidence
}

Anne Hawker

RI and Ministry of Social Development, New Zealand. Anne.hawker011@msd.govt.nz

The United Nations Convention on the Rights of People with disabilities has driven changed expectations on the way in which States are expected to relate to disabled people and assist in building an inclusive society.

The World Report on Disabilities has provided both statistical information and examples of best practice including in the employment space.

In the employment area, states are expected to provide a leadership role and provide support for private enterprise. Taking a much more partnership approach to the employment of people with disabilities has increased understanding of respective needs and helped shape different conversations. These conversations have been informed by research in to what employers perceive as barriers to employing disabled people. Providing employers with answers to their concerns has provided them with the confidence to employ and retain employees with disabilities. The mainstream approach has moved the discussion away from differences to an understanding that in many situations, this is no different to what is already provided to other employees. Confident employers are likely to provide repeat opportunities and also be good role models for other employers.

The world financial crisis has sharpened enterprises understanding of the economic impact of failing to take a proactive approach to their employees. Responsibility for building knowledge is equally shared by the employee and the employer. The employee's direct line manager is has a crucial role in building connectedness for those for whom they have managerial oversight.

Demedicalising the way people with disabilities are either retained or enter the workforce will be crucial in building the confidence of employers. Language and culture play an important part in building a connected and confident workplace. 